\title{
Correction to: Personalized Travel Recommendation via Multi-view Representation Learning
}

Yujun Zhang, Bing Han, Xinbo Gao, and Haoran Li

\section{Correction to: \\ Chapter "Personalized Travel Recommendation via Multi-view Representation Learning" in: Z. Lin et al. (Eds.): Pattern Recognition and Computer Vision, LNCS 11859, https://doi.org/10.1007/978-3-030-31726-3_9}

In the original version of the paper the author's name was incorrectly spelled as Bin Han. It has been corrected to Bing Han.

Furthermore the foundation numbers were missing in the footnote:

The full funding information is:

This work was supported by the National Natural Science Foundation of China (41831072;61572384; 61603233), China's postdoctoral fund first-class funding (2014M560752), Shannxi province postdoctoral science fund Key Research and Development Program of Shaanxi Province (2017KW-017). 